\title{
Mitochondrial dysfunction and activation of iNOS are responsible for the palmitate-induced decrease in adiponectin synthesis in 3T3L1 adipocytes
}

\author{
Min Jae Jeon ${ }^{1 *}$, Jaechan Leem ${ }^{2 *}$, \\ Myoung Seok Ko', Jung Eun Jang ${ }^{2}$, \\ Hye-Sun Park ${ }^{1}$, Hyun Sik Kim ${ }^{1}$, \\ Mina Kim ${ }^{1}$, Eun Hee Kim², Hyun Ju Yoo', \\ Chul-Ho Lee ${ }^{3}$, In-Sun Park ${ }^{4}$, Ki-Up Lee ${ }^{2}$ \\ and Eun Hee Koh ${ }^{2,5}$ \\ ${ }^{1}$ Asan Institute for Life Sciences \\ Seoul 138-736, Korea \\ ${ }^{2}$ Department of Internal Medicine \\ University of Ulsan College of Medicine \\ Seoul 138-736, Korea \\ ${ }^{3}$ Korea Research Institute of Bioscience and Biotechnology \\ Daejeon 305-333, Korea \\ ${ }^{4}$ Department of Anatomy \\ College of Medicine \\ Inha University \\ Incheon 401-103, Korea \\ ${ }^{5}$ Corresponding author: Tel, 82-2-3010-3248; \\ Fax, 82-2-3010-6962; E-mail, ehk@amc.seoul.kr \\ *These authors contributed equally to this work. \\ http://dx.doi.org/10.3858/emm.2012.44.9.064
}

Accepted 17 July 2012

Available Online 19 July 2012

Abbreviations: ATF3, activating transcription factor 3; CCCP, carbonyl cyanide m-chlorophenyl hydrazone; CHOP, CCAAT/ enhancer-binding protein; eNOS, endothelial nitric oxide synthase; ER, endoplasmic reticulum; ETC, electron transport chain; FFA, free fatty acid; JNK, c-Jun N-terminal kinases; iNOS, inducible nitric oxide synthase; mtDNA, mitochondrial DNA; mtTFA, mitochondrial transcription factor $A$; NO, nitric oxide; NRF-1, nuclear respiratory factor-1; PPAR $\gamma$, peroxisome proliferator-activated receptor- $\gamma$; ROS, reactive oxygen species; SMT, S-methylisothiourea sulfate; TNF- $\alpha$, tumor necrosis factor- $\alpha$; XBP1, X-box binding protein 1

\section{Abstract \\ Mitochondrial dysfunction and endoplasmic retic- ulum (ER) stress are considered the key determinants}

of insulin resistance. Impaired mitochondrial function in obese animals was shown to induce the ER stress response, resulting in reduced adiponectin synthesis in adipocytes. The expression of inducible nitric oxide synthase (iNOS) is increased in adipose tissues in genetic and dietary models of obesity. In this study, we examined whether activation of iNOS is responsible for palmitate-induced mitochondrial dysfunction, ER stress, and decreased adiponectin synthesis in 3T3L1 adipocytes. As expected, palmitate increased the expression levels of iNOS and ER stress response markers, and decreased mitochondrial contents. Treatment with iNOS inhibitor increased adiponectin synthesis and reversed the palmitate-induced ER stress response. However, the iNOS inhibitor did not affect the palmitate-induced decrease in mitochondrial contents. Chemicals that inhibit mitochondrial function increased iNOS expression and the ER stress response, whereas measures that increase mitochondrial biogenesis (rosiglitazone and adenoviral overexpression of nuclear respiratory factor-1) reversed them. Inhibition of mitochondrial biogenesis prevented the rosiglitazone-induced decrease in iNOS expression and increase in adiponectin synthesis. These results suggest that palmitate-induced mitochondrial dysfunction is the primary event that leads to iNOS induction, ER stress, and decreased adiponectin synthesis in cultured adipocytes.

Keywords: adiponectin; endoplasmic reticulum stress; inducible nitric oxide synthase; mitochondrial dysfunction; nitric oxide

\section{Introduction}

Adipose tissue is an important endocrine organ that secretes many bioactive substances (adipocytokines). One of these, adiponectin, has many favorable effects on metabolism, including improvement of insulin action and reduction of atherosclerotic processes (Kadowaki et al., 2006; Koh et al., 2007). Unlike other adipocytokines, the plasma 
level of adiponectin is reduced in obese individuals (Kern et al., 2003; Scherer, 2006). We previously reported that mitochondrial function is essential for adiponectin synthesis in adipocytes, and that mitochondrial dysfunction in adipose tissue explains decreased adiponectin synthesis in obesity (Koh et al., 2007). Of particular interest in this study was the observation that impaired mitochondrial function activates a series of mechanisms involving endoplasmic reticulum (ER) stress, c-Jun $\mathrm{N}$-terminal kinases (JNK), and activating transcription factor 3 (ATF3) to decrease adiponectin synthesis.

Nitric oxide (NO) is produced from L-arginine by the enzymatic action of NADPH-dependent NO synthases (NOS) (Chung et al., 2012). Among the known isoforms of NOS, activation of endothelial NOS (eNOS) is necessary for mitochondrial biogenesis (Nisoli et al., 2003) and adiponectin synthesis (Koh et al., 2010). Inducible NOS (iNOS) is also expressed in adipose tissue (Elizalde et al., 2000), and iNOS expression is increased in adipose tissues in genetic and dietary models of obesity (Perreault and Marette, 2001). In addition, obese mice lacking iNOS are sensitized to the metabolic actions of rosiglitazone, a peroxisome proliferator-activated receptor- $\gamma$ (PPAR $\gamma$ ) agonist, that result in increased plasma adiponectin levels (Dallaire et al., 2008).

Plasma levels of free fatty acids (FFAs) are increased in obese individuals and those with type 2 diabetes (Boden, 1997). FFAs have been shown to cause mitochondrial dysfunction and ER stress (Guo et al., 2007; Rachek et al., 2007). In particular, saturated fatty acids, i.e., palmitate and stearate, induce inflammation and insulin resistance in adipose tissue (Kennedy et al., 2009), whereas polyunsaturated fatty acids reduce body weight and insulin resistance (Kalupahana et al., 2010; Sato et al., 2010). Palmitate also decreases adiponectin synthesis (Xi et al., 2007) and increases iNOS expression in adipocytes (McCall et al., 2010).

In this study, we investigated the role of iNOS activation in the palmitate-induced decrease in adiponectin synthesis. As excessive NO produced by iNOS was shown to increase mitochondrial damage (Holohan et al., 2008), we hypothesized that palmitate-induced activation of iNOS would be primarily responsible for mitochondrial dysfunction and ER stress, and the consequent decrease in adiponectin synthesis. As expected, palmitate-induced iNOS expression was responsible for ER stress and decreased adiponectin synthesis. However, iNOS did not affect mitochondrial contents, and conversely, palmitate-induced mitochondrial
A

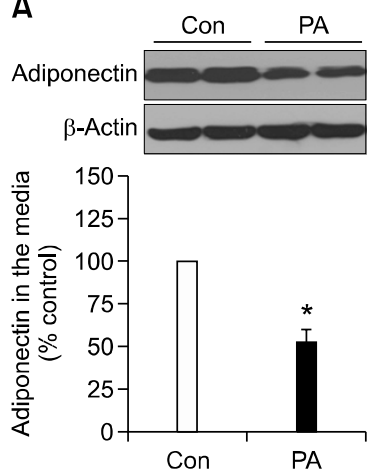

B

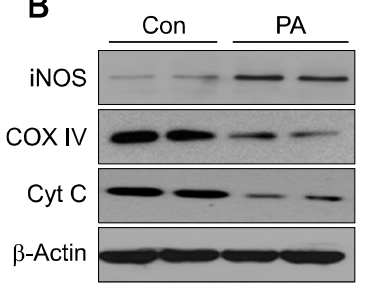

C

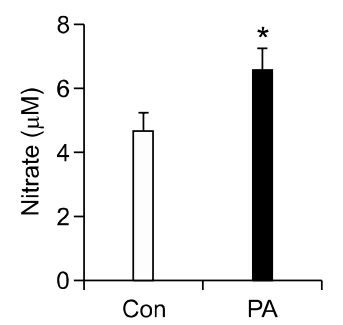

D

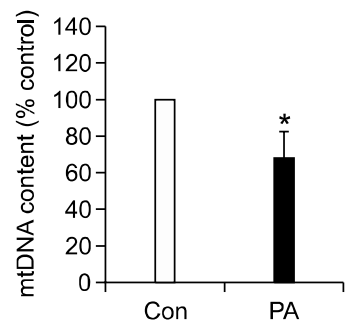

E

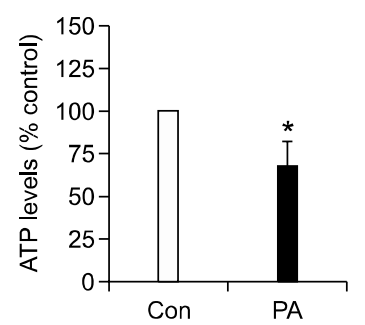

Figure 1. Effect of palmitate on adiponectin synthesis and iNOS expression in cultured adpocytes. Palmitate solutions containing $2 \%$ fatty acid-free bovine serum albumin were prepared in DMEM containing no serum. Differentiated 3T3L1 adipocytes were treated with palmitate (PA, $500 \mu \mathrm{M})$ for 24 hours. A 2\% BSA control was included with or without palmitate. (A) Representative western blots of adiponectin expression in the cells and adiponectin levels in the culture media. (B) Effects of palmitate on the expression levels of iNOS and mitochondrial proteins in 3T3L1 adipocytes. COX IV, cytochrome C oxidase subunit II; Cyt C, cytochrome $C$. (C) Nitrate levels in the culture media, measured using the Griess Reagent System. (D) mtDNA contents measured by real-time PCR analysis. (E) Intracellular ATP levels measured by ATP fluorometric assay kit. (F) Intracellular ROS levels in response to PA treatment. Data in $A, C, D, E$ and $F$ are shown as means \pm SEM ( $n=5$ each). ${ }^{*} P<$ 0.05 versus untreated cells.

dysfunction was responsible for iNOS induction.

\section{Results}

Palmitate decreases adiponectin synthesis and increases iNOS expression in cultured adipocytes

As reported previously (Xi et al., 2007), palmitate 

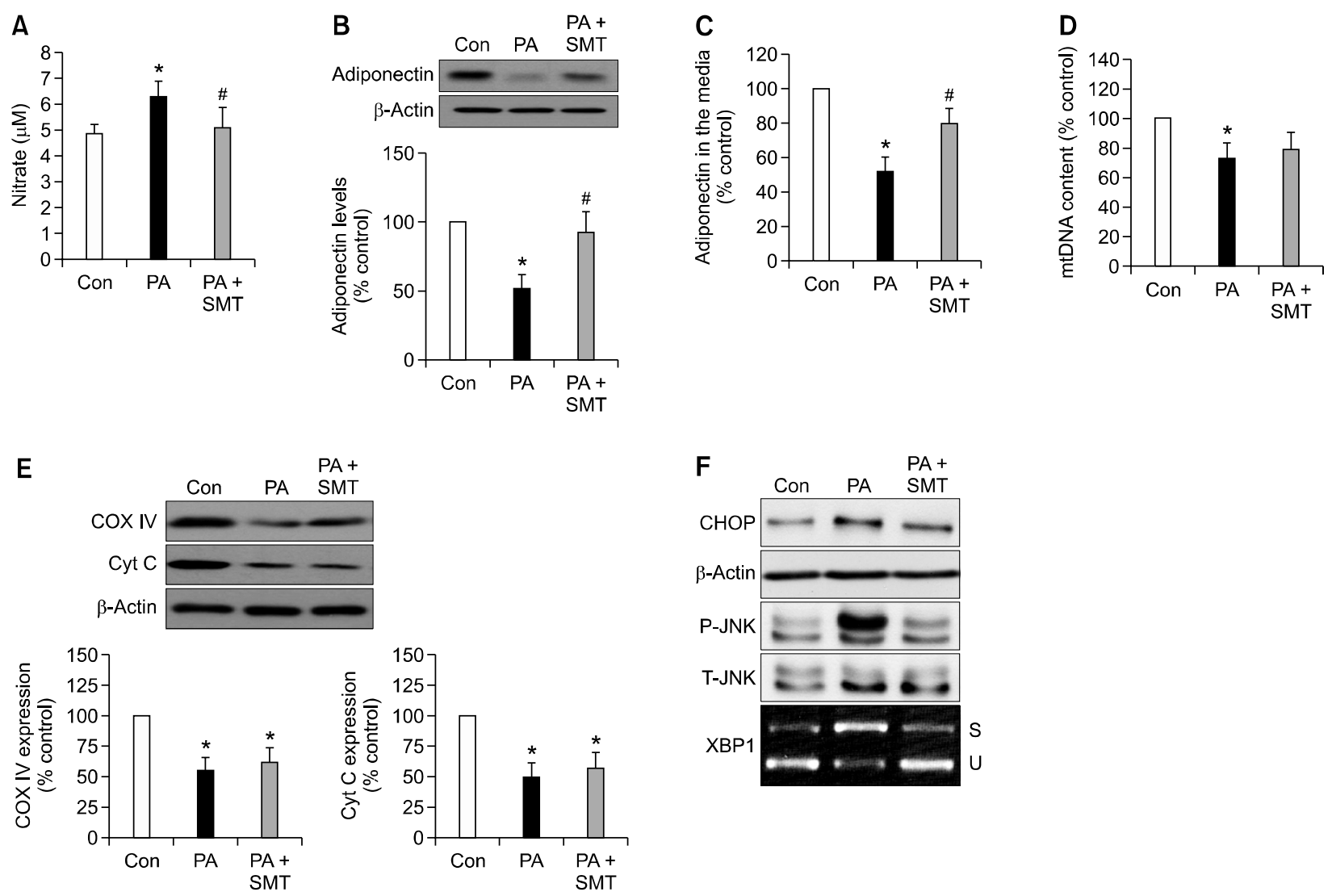

Figure 2. iNOS inhibition by SMT reverses the inhibitory effect of palmitate on the expression of adiponectin. Differentiated 3T3L1 adipocytes were serum-deprived and cultured for 24 hours in the absence or presence of palmitate (PA, $500 \mu \mathrm{M}$ ) alone or in combination with SMT (30 $\mu \mathrm{M})$. (A) Nitrate levels in the culture media. (B, C) Effect of iNOS inhibition on adiponectin synthesis. Representative Western blots and band density of adiponectin expression in the cells (B) and adiponectin levels in the culture media (C). (D, E) SMT does not affect mitochondrial contents. (D) mtDNA contents measured by real-time PCR analysis. (E) Representative Western blots and band density of mitochondrial proteins. (F) Effect of SMT on the ER stress response. Representative Western blots of CHOP and pJNK, and RT-PCR analysis of spliced XBP1 (S) and unspliced XBP1 (U) mRNA. Data in panels A-E are shown as means \pm SEM ( $n=5$ each). ${ }^{*} P<0.05$ versus untreated cells; ${ }^{*} P<0.05$ versus palmitate-treated cells.

significantly decreased adiponectin expression in 3T3L1 adipocytes and its secretion into culture media (Figure 1A). Additionally, in agreement with a previous study (McCall et al., 2010), palmitate increased iNOS expression in cultured adipocytes (Figure 1B), and this was associated with increased NO synthesis by adipocytes as measured by the nitrite concentration in the media (Figure 1C). Palmitate decreased mitochondrial DNA (mtDNA) contents (Figure 1D) and the expression of mitochondrial respiratory proteins cytochrome $\mathrm{C}$ oxidase and cytochrome C (Figure 1B). Palmitate also decreased ATP levels and increased reactive oxygen species (ROS) generation (Figures 1E and F).

\section{Inhibition of iNOS reverses palmitate-induced ER stress response but does not affect mitochondrial contents}

Excessive NO is known to inhibit electron flow in the mitochondrial electron transport chain (ETC) and to increase ROS production and mitochondrial damage (Cassina and Radi, 1996). We therefore reasoned that iNOS inhibition would recover palmitate-induced decreases in mtDNA contents and mitochondrial protein expression. As expected, S-methylisothiourea sulfate (SMT), an iNOS-specific inhibitor, inhibited NO synthesis (Figure 2A), and recovered the palmitate-induced decrease in adiponectin expression (Figure 2B) and secretion into media (Figure 2C). However, contrary to our expectation, treatment with SMT did not affect mitochondrial DNA content (Figure 2D) or the expression of mitochondrial proteins (Figure 2E).

As previously reported (McCall et al., 2010), palmitate significantly increased the levels of ER 

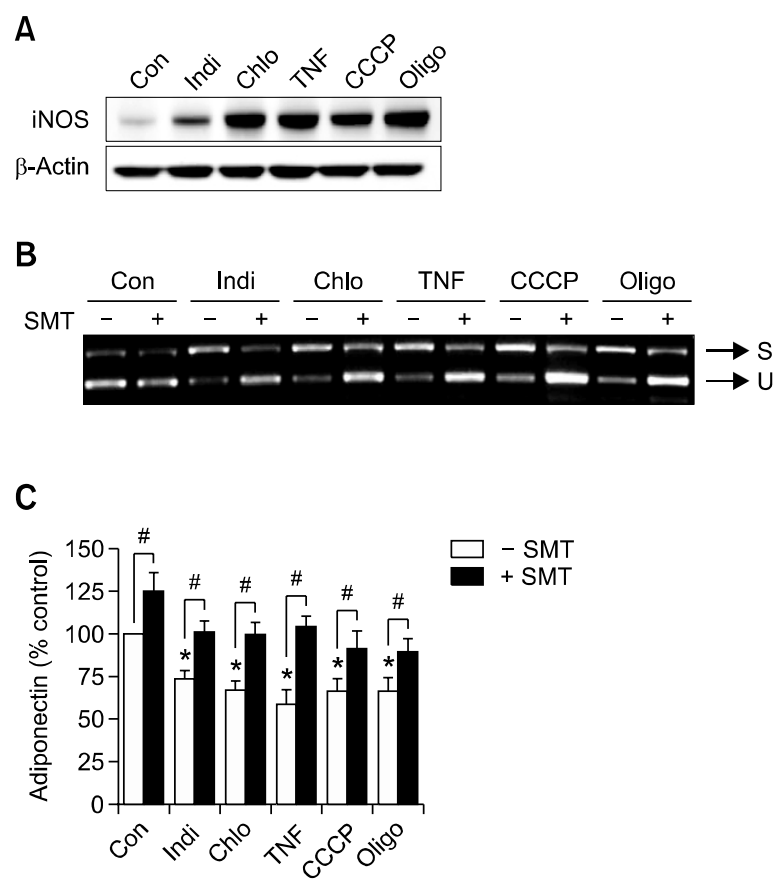

Figure 3. iNOS activation is responsible for ER stress induced by mitochondrial inhibitors. (A) Effect of mitochondrial inhibitors on iNOS expression in 3T3L1 adipocytes. Differentiated 3T3L1 adipocytes were treated with idinavir (Indi, $100 \mu \mathrm{M}$ ), chloramphenicol (Chlo, $100 \mu \mathrm{g} / \mathrm{ml}$ ), TNF- $\alpha$ (TNF, $10 \mathrm{ng} / \mathrm{ml}), C C C P(0.4 \mu \mathrm{g} / \mathrm{ml})$, and oligomycin (Oligo, 10 $\mu \mathrm{g} / \mathrm{ml}$ ) for 8 hours. (B, C) Effect of iNOS inhibition on ER stress and adiponectin synthesis. RT-PCR analysis of spliced XBP1 (S) and unspliced XBP1 (U) mRNA in the cells (B) and adiponectin levels in the media (C). Cells were co-treated with or without SMT $(30 \mu \mathrm{M})$ and mitochondrial inhibitors ( $n=6$ per group). Data are shown as means \pm SEM. ${ }^{*} P<$ 0.05 versus untreated cells; ${ }^{\sharp} P<0.05$ versus chemicals-untreated cells.

stress response markers (anti-CCAAT/enhancer-binding protein (CHOP) and X-box binding protein 1 (XBP1) splicing) and the phosphorylation of JNK, a downstream signaling molecule of the ER stress response shown to be involved in adiponectin synthesis (Koh et al., 2007) in cultured adipocytes (Figure 2F). Inhibition of iNOS by SMT nearly completely reversed palmitate-induced changes in the ER stress response and JNK. Taken together, these results suggest that palmitate-induced activation of iNOS is responsible for the ER stress response and decreased adiponectin synthesis in cultured adipocytes, but is not responsible for changes in mitochondrial contents.

\section{Mitochondrial dysfunction decreases adiponectin synthesis by increasing iNOS expression}

We previously showed that various chemicals that inhibit mitochondrial function [indinavir, chloramphenicol, tumor necrosis factor- $\alpha$ (TNF- $\alpha$ ), carbonyl cyanide m-chlorophenyl hydrazone (CCCP), and oligomycin] reduce adiponectin synthesis, and increase the ER stress response (Koh et al., 2007). To determine whether this effect is mediated by iNOS, we examined the effects of these chemicals on iNOS expression. iNOS expression was significantly increased by these chemicals (Figure $3 \mathrm{~A}$ ). SMT significantly decreased XBP1 splicing induced by these chemicals (Figure 3B) and increased adiponectin synthesis (Figure $3 \mathrm{C}$ ).

\section{Increased mitochondrial biogenesis decreases iNOS expression in cultured adipocytes}

We next questioned whether the palmitate-induced increase in iNOS expression is mediated by mitochondrial dysfunction. Increased mitochondrial biogenesis improves mitochondrial function (Spiegelman, 2007; Wang et al., 2010), and rosiglitazone increases adiponectin synthesis in adipocytes by increasing mitochondrial biogenesis (Koh et al., 2007). In this study, we examined the effects of mtTFA siRNA on rosiglitazone-induced changes in iNOS expression. As expected (Wilson-Fritch et al., 2004; Koh et al., 2007), rosiglitazone increased adiponectin secretion, and this was accompanied by a decrease in the expression of iNOS in cultured 3T3L1 adipocytes (Figures 4B and 4C). These effects of rosiglitazone were significantly lower in cells pre-treated with siRNA against mitochondrial transcription factor A (mtTFA), a mitochondrial matrix protein essential for the replication and transcription of mtDNA (Kelly and Scarpullar, 2004) (Figures 4A-4C). To further confirm the reducing effect of increased mitochondrial biogenesis on iNOS expression, we examined the effect of nuclear respiratory factor-1 (NRF-1) overexpression. NRF-1 is a transcription factor that regulates the expression of nuclear-encoded mitochondrial genes, including mtTFA. Adenovirus-mediated overexpression of NRF-1 (Ad-NRF-1) decreased iNOS expression in the presence of palmitate, and this was accompanied by an increase in adiponectin synthesis (Figures 4D and 4E). siRNA against mtTFA reversed the effects of Ad-NRF-1 on iNOS expression and adiponectin synthesis (Figures 4D and 4E). Together, these results suggest that mitochondrial dysfunction induces ER stress by increasing iNOS expression in adipocytes, and that improved mitochondrial function by mitochondrial biogenesis reduces iNOS expression and increases adiponectin synthesis.

\section{Discussion}

Accumulating evidence indicates that NO produ- 
A

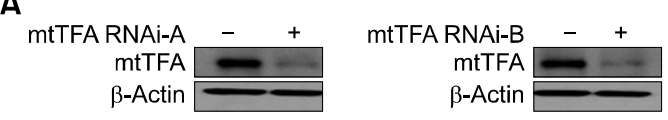

B

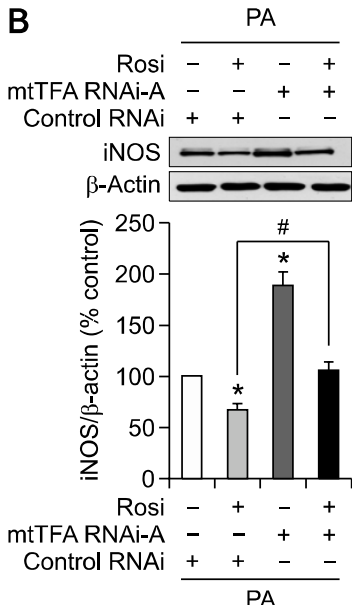

C

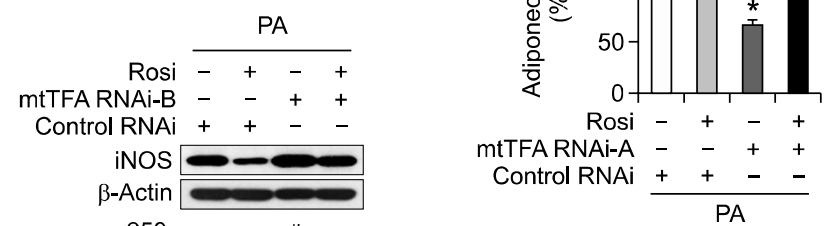

D
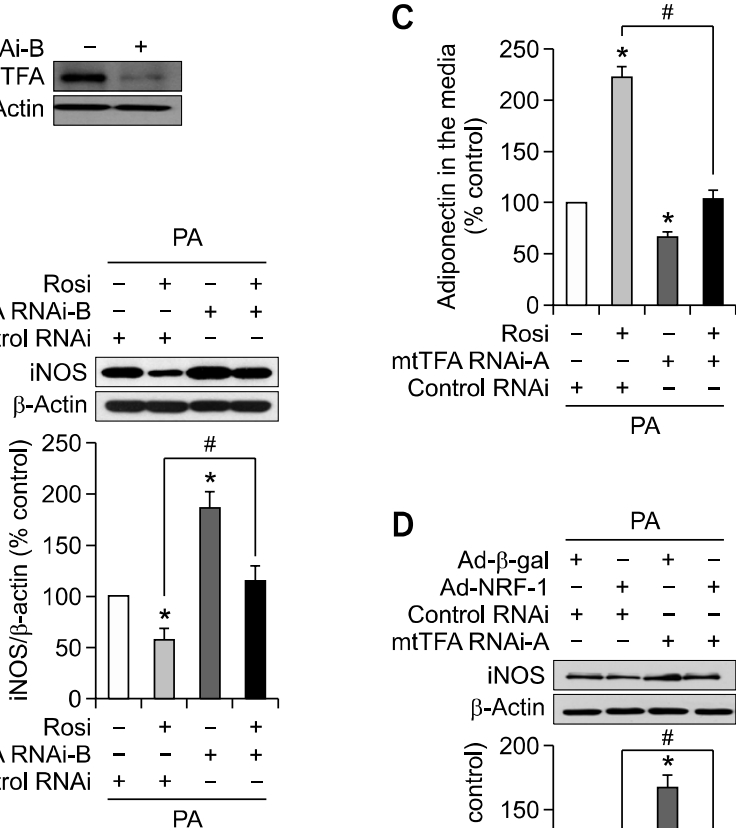

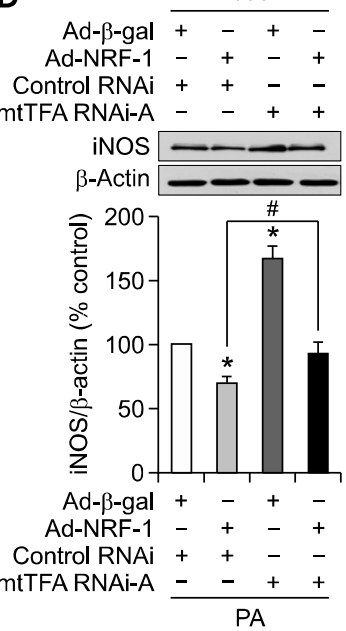

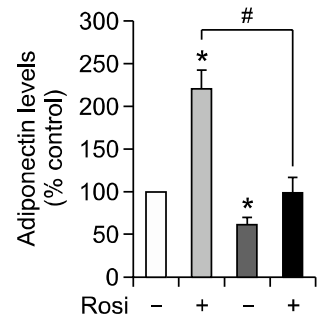

mTTFA RNAi-B - - + +

Control RNAi $++-\quad$

E

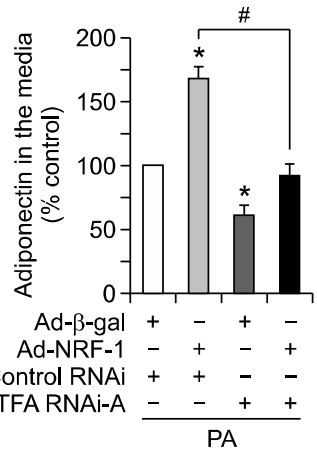

Figure 4. Increased mitochondrial biogenesis decreases iNOS expression and increases adiponectin synthesis. (A-C) The effect of mtTFA siRNA on the rosiglitazone-induced decrease in iNOS expression. Two siRNAs targeting different sequences of mtTFA were made. 3T3L1 preadipocytes were transfected with 100 pmol of each mtTFA siRNA (mtTFA RNAi-A or B) or control siRNAs (Control RNAi), and then differentiated into mature adipocytes. The differentiated cells were treated with $500 \mu \mathrm{M}$ palmitate (PA) with or without rosiglitazone (Rosi, $20 \mu \mathrm{M}$ ) for 24 hours. (A) Westen blot was used to confirm siRNA's target gene knockdown effect. (B) iNOS protein expression. (C) Adiponectin levels in culture media. Data are presented as mean \pm SEM $(n=5$ each). ${ }^{*} P<0.05$ versus control (with PA treatment); ${ }^{P} P<0.05$ versus control siRNA-transfected cells. (D, E) The effect of mtTFA siRNA on Ad-NRF-1-induced decrease in iNOS expression. 3T3L1 preadipocytes were transfected with 100 pmol mtTFA siRNA (mtTFA RNAi) or control siRNA. Following this, 3T3L1 preadipocytes were infected with adenoviruses carrying $\beta$-gal (Ad- $\beta$-gal) or NRF-1 (Ad-NRF-1) at a titer of $5 \times 10^{6}$ pfu/ml for 1 hour at $37^{\circ} \mathrm{C}$ without serum. $3 T 3 \mathrm{~L} 1$ preadipocytes were then differentiated into mature adipocytes, and the differentiated cells were treated with $500 \mu \mathrm{M}$ palmitate for 24 hours. (D) Representative Western blots and relative band densities of iNOS. (E) Adiponectin levels in the culture media. ${ }^{*} P<0.05$ versus Ad- $\beta$-gal-transfected cells; ${ }^{P} P<0.05$ versus control siRNA-transfected cells.

ced by NOS plays a crucial role in energy metabolism (Kapur et al., 2000). Among the known types of NOS, iNOS produces high concentrations of NO in response to various inflammatory signals (Nathan and Xie, 1994). iNOS is also expressed in tissues other than inflamed tissues, and its expression is increased in adipose tissue and muscle in animal models of dietary and genetic obesity (Zhou et al., 2000; Perreault et al., 2001). iNOS-/- mice are protected from high fat-induced insulin resistance (Perreault et al., 2001).

Mitochondrial function was shown to be essential for adiponectin synthesis in adipocytes (Koh et al., 2007). In the present study, we showed that palmitate-induced mitochondrial dysfunction induced
iNOS expression, and that this was responsible for ER stress and reduced adiponectin synthesis in cultured 3T3L1 adipocytes. Our results are consistent with those of a previous study, which showed that excessive NO downregulates the gene expression and secretion of adiponectin (Nozaki et al., 2007). A recent study also showed that the effect of rosiglitazone on adiponectin levels was markedly increased in iNOS-/- mice (Dallaire et al., 2008).

Excessive NO in the cell has been shown to increase mitochondrial damage and to cause mitochondrial dysfunction by inhibiting mitochondrial ETC (Cassina and Radi, 1996). We therefore reasoned that iNOS inhibitor would recover palmitate- 
induced decreases in mtDNA and mitochondrial protein expression. As expected (Crunkhorn et al., 2007; Rachek et al., 2007), palmitate significantly decreased mtDNA content and mitochondrial protein levels. However, iNOS inhibition by SMT did not increase mtDNA content or the expression of mitochondrial respiratory proteins. In our study, chemicals that cause mitochondrial dysfunction induced iNOS expression in cultured adipocytes. Although this particular experiment has not been performed before, this is consistent with previous studies (Yang et al., 2002; Lee and Wei, 2005).

In this study, we found that rosiglitazone, a PPAR $\gamma$ agonist, decreased iNOS expression in cultured adipocytes. This finding is consistent with previous studies: various PPAR $\gamma$ agonists have been shown to inhibit iNOS expression in islet cells (Abaraviciene et al., 2008), myocardium (Liu et al., 2004), lung (Cuzzocrea et al., 2004), and 3T3L1 adipocytes, and in the epididymal fat of Otsuka Long-Evans Tokushima Fatty rats (Linscheid et al., 2003). PPAR $\gamma$ agonists increase mitochondrial biogenesis (Wilson-Fritch et al., 2004; Koh et al., 2007), and increased mitochondrial biogenesis is known to improve mitochondrial function (Wenz et al., 2008). Our data, which indicate that rosiglitazone and Ad-NRF-1 reversed the palmitate-induced increase in iNOS expression, and that treatment with siRNA against mtTFA reversed the rosiglitazone- and Ad-NRF-1-induced decreases in iNOS expression, suggest that palmitate-induced mitochondrial dysfunction is responsible for iNOS induction.

Palmitate was shown to induce iNOS production (Tsang et al., 2004), mitochondrial dysfunction (Yuzefovych et al., 2010) and ER stress (Green and Olson, 2011). The novelty of our study is that we showed the sequence of these events. We provided new evidence that mitochondrial dysfunction is the primary cause of iNOS induction, ER stress response and decreased adiponectin synthesis (Supplemental Figure S1). However, excessive NO in the cell is known to increase mitochondrial damage and to cause mitochondrial dysfunction by inhibiting mitochondrial electron-transfer complexes (Cassina and Radi, 1996). This effect of NO may help the cells withstand severe stress by reducing intracellular ROS generation (Poliandri et al., 2006). However, it may also result in a vicious cycle, in which mitochondrial dysfunction leads to iNOS induction, NO increases, and further mitochondrial dysfunction.

In summary, we have demonstrated that palmitate-induced mitochondrial dysfunction induces iNOS expression and the ER stress response, and reduces adiponectin synthesis in cultured adipo- cytes. Increases in mitochondrial biogenesis by Ad-NRF-1 or rosiglitazone decreased iNOS expression and ER stress, and this was reversed by siRNA against mtTFA. The iNOS inhibitor SMT increased adiponectin synthesis by reducing ER stress, but did not affect mitochondrial contents. Taken together, these results suggest that palmitate-induced mitochondrial dysfunction is the primary event that leads to iNOS induction, ER stress, and decreased adiponectin synthesis in cultured adipocytes. Excessive NO produced by iNOS links mitochondrial dysfunction to ER stress and decreased adiponectin synthesis.

\section{Methods}

\section{Cell culture}

3T3L1 fibroblasts were cultured in Dulbecco's modified Eagle's medium (DMEM) supplemented with $10 \%$ fetal bovine serum in an incubator with $5 \% \mathrm{CO}_{2} / 95 \% \mathrm{O}_{2}$ at $37^{\circ} \mathrm{C}$. 3T3L1 pre-adipocytes were differentiated to mature adipocytes by a conventional method (Koh et al., 2007) and were maintained as subconfluent cultures.

\section{Measurement of adiponectin concentration}

Adiponectin concentrations in the culture media were measured by an enzyme-linked immunosorbent assay (ELISA) kit (Alpco Diagnostics, Salem, NH) according to the manufacturer's instructions.

\section{Measurement of nitrite concentration}

Nitrite concentrations in the culture media were measured by the Total Nitric Oxide Assay kit (Assay Designs, Ann Arbor, MI) according to the manufacturer's instructions.

\section{Measurement of ROS levels}

Intracellular ROS generation was measured by flow cytometry using DCFH2-DA (Molecular Probes, Eugene, $\mathrm{OR}$ ). For measurement of intracellular ROS levels, cells were incubated for $15 \mathrm{~min}$ with $2.5 \mu \mathrm{mol} / \mathrm{ml} \mathrm{DCFH2-DA}$ at $37^{\circ} \mathrm{C}$ for $30 \mathrm{~min}$. The increase in DCFH2-DA oxidation was measured by a flow cytometry (FACSCalibur, San Jose, $\mathrm{CA}$ ). Fluorescence was measured at an excitation wavelength of $488 \mathrm{~nm}$ and an emission wavelength of $530 \mathrm{~nm}$.

\section{Measurement of intracellular ATP levels}

Intracellular ATP levels were measured using an ATP determination kit (BioVision, Mountain View, CA) according to manufacturer's instructions.

\section{Western blot analysis}

Differentiated 3T3L1 cells were lysed in a buffer containing $20 \mathrm{mM}$ Tris- $\mathrm{HCl}$ (pH 7.4), 1 mM EDTA, $140 \mathrm{mM} \mathrm{NaCl}, 1 \%$ 
NP-40, $1 \mathrm{mM} \mathrm{Na} \mathrm{VO}_{4}, 1 \mathrm{mM}$ phenylmethylsulfonyl fluoride, $50 \mathrm{mM} \mathrm{NaF}$, and $10 \mu \mathrm{g} / \mathrm{ml}$ aprotinin. The protein $(40-50 \mu \mathrm{g})$ was subjected to immunoblotting with specific antibodies. Antibodies against phospho-JNK and cytochrome C oxidase were from Cell Signaling Technology (Beverly, MA). Antibodies against CHOP and mtTFA antibody were from Santa Cruz Biotechnology (Santa Cruz, CA). Anti-iNOS, anti-cytochrome $C$ antibody, and anti- $\beta$-actin were from BD Biosciences (San Jose, CA) and Sigma-Aldrich (St Louis, $\mathrm{MO})$, respectively.

\section{Analysis of spliced XBP1 mRNA}

XBP1 is a critical regulator of ER function and the ER stress response (Hotamisligil, 2006). Upon activation of the unfolded protein response, XBP1 mRNA is spliced by IRE1 to remove a 26-nucleotide intron and generate a translational frame shift. The resulting spliced mRNA (active form) encodes a protein with a novel carboxy-terminus that acts as a potent transcriptional activator. The target segment of the XBP1 cDNA was amplified by polymerase chain reaction (PCR) amplication. After separating the restriction digests, the cDNA fragments were resolved on $2 \%$ agarose gel. XBP1 splicing was measured by PCR using the following primers: forward, 5'-AAACAGAGTAGCAGCGCA GACTGC-3', reverse, 5'-GGATCTCTAAAACTAGAGGCTT GGTG-3'. The PCR procedure included an initial incubation for 3 minutes at $92^{\circ} \mathrm{C}$, followed by 40 cycles of 45 seconds at $92^{\circ} \mathrm{C}, 45$ seconds at $52^{\circ} \mathrm{C}$, and 45 seconds at $72^{\circ} \mathrm{C}$, and a final incubation for 10 minutes at $72^{\circ} \mathrm{C}$. The amplified fragment was then digested by the restriction endonuclease Pstl for 2 hours at $37^{\circ} \mathrm{C}$ to reveal a restriction site that is lost after IRE1-mediated cleavage and splicing of the mRNA. After separating the restriction digests, the cDNA fragments were resolved on a $2 \%$ agarose gel.

\section{Small interfering RNA (siRNA)}

We designed two different siRNAs targeting different sequences of mtTFA: 5'-GUACAUUUUGGGAAAGUAA-3' and 5'-GUCACUGACAAGUUUGCAAdTdT-3'. We also made control siRNAs, which have the same GC content as the target sequences and do not cause silencing of gene expression (Bioneer, Daejon, Korea). Aliquots of $1.5 \times 10^{5}$ cells were seeded into each well of 6 -well plates and transfected with 100 pmol siRNA using lipofectamine (Invitrogen, Carlsbad, CA) according to the manufacturer's instructions.

\section{Quantification of mtDNA content}

mtDNA contents were quantified by real-time PCR on an ABI PRISM 7000 sequence detection system (Applied Biosystems, Foster City, CA) using a SYBR Green kit (Applied Biosystems) according to the manufacturer's protocol. Mouse nuclear 18S rRNA was used as the internal control. The ratio of the expression level of the target gene to that of the 18S rRNA was calculated by subtracting the Ct (threshold cycle) value of the target gene from the Ct value of $18 \mathrm{~S}$ rRNA. Primers for detecting the cytochrome b gene $(14,146-15,289)$ from the murine mitochondrial genome were designed on the basis of the GenBank nucleotide sequence. The primer sequences were: forward, 5'- CCA CTT CAT CTT ACC ATT TA -3'; reverse, 5'ATC TGC ATC TGA GTT TAA TC-3' (GenBank AB042432.1, Mus musculus domesticus mitochondrion) (Koh et al., 2007).

\section{Recombinant adenovirus}

NRF-1 adenovirus was a kind gift from Prof. Y. K. Pak (Kyung Hee University, Seoul, Korea).

\section{Statistical analysis}

All values are given as mean \pm SEM. Differences between two groups were assessed using unpaired two-tailed $t$-test. Data from more than two groups were assessed by analysis of variance (ANOVA) followed by a post-hoc least significant difference test. $P<0.05$ was considered statistically significant.

\section{Supplemental data}

Supplemental data include a figure and can be found with this article online at http://e-emm.or.kr/article/article_files/ SP-44-9-06.pdf.

\section{Acknowledgements}

This work was supported by a National Research Foundation grant funded by the Korean government, Ministry of Education, Science, and Technology (2009-0091988 to KUL).

\section{References}

Abaraviciene SM, Lundquist I, Salehi A. Rosiglitazone counteracts palmitate-induced beta-cell dysfunction by suppression of MAP kinase, inducible nitric oxide synthase and caspase 3 activities. Cell Mol Life Sci 2008;65:2256-65

Boden $\mathrm{G}$. Role of fatty acids in the pathogenesis of insulin resistance and NIDDM. Diabetes 1997;46:3-10

Cassina A, Radi R. Differential inhibitory action of nitric oxide and peroxynitrite on mitochondrial electron transport. Arch Biochem Biophys 1996;328:309-16

Chung BH, Kim S, Kim JD, Lee JJ, Baek YY, Jeoung D, Lee $\mathrm{H}$, Choe J, Ha KS, Won MH, Kwon YG, Kim YM. Syringaresinol causes vasorelaxation by elevating nitric oxide production through the phosphorylation and dimerization of endothelial nitric oxide synthase. Exp Mol Med 2012;44:191-201

Crunkhorn S, Dearie F, Mantzoros C, Gami H, da Silva WS, Espinoza D, Faucette R, Barry K, Bianco AC, Patti ME. Peroxisome proliferator activator receptor gamma coactivator-1 expression is reduced in obesity: potential pathogenic role of saturated fatty acids and p38 mitogen-activated protein kinase activation. J Biol Chem 2007;282:15439-50

Cuzzocrea S, Pisano B, Dugo L, lanaro A, Maffia P, Patel NS, 
Di Paola R, lalenti A, Genovese T, Chatteriee PK, Di Rosa M, Caputi AP, Thiemermann C. Rosiglitazone, a ligand of the peroxisome proliferator-activated receptor-gamma, reduces acute inflammation. Eur J Pharmacol 2004;483:79-93

Dallaire P, Bellmann K, Laplante M, Gelinas S, CentenoBaez C, Penfornis P, Peyot ML, Latour MG, Lamontagne J, Trujillo ME, Scherer PE, Prentki M, Deshaies Y, Marette A. Obese mice lacking inducible nitric oxide synthase are sensitized to the metabolic actions of peroxisome proliferator-activated receptor-gamma agonism. Diabetes 2008; 57:1999-2011

Elizalde $M$, Ryden $M$, van Harmelen $V$, Eneroth $P$, Gyllenhammar H, Holm C, Ramel S, Olund A, Arner P, Andersson K. Expression of nitric oxide synthases in subcutaneous adipose tissue of nonobese and obese humans. J Lipid Res 2000;41:1244-51

Green CD, Olson LK. Modulation of palmitate-induced endoplasmic reticulum stress and apoptosis in pancreatic beta-cells by stearoyl-CoA desaturase and Elovi6. Am J Physiol Endocrinol Metab 2011;300:E640-9

Guo W, Wong S, Xie W, Lei T, Luo Z. Palmitate modulates intracellular signaling, induces endoplasmic reticulum stress, and causes apoptosis in mouse 3T3-L1 and rat primary preadipocytes. Am J Physiol Endocrinol Metab 2007;293:E576-86

Holohan C, Szegezdi E, Ritter T, O'Brien T, Samali A. Cytokine-induced beta-cell apoptosis is NO-dependent, mitochondria-mediated and inhibited by BCL-XL. J Cell Mol Med 2008;12:591-606

Hotamisligil GS. Inflammation and metabolic disorders. Nature 2006;444:860-7

Kadowaki T, Yamauchi T, Kubota N, Hara K, Ueki K, Tobe K. Adiponectin and adiponectin receptors in insulin resistance, diabetes, and the metabolic syndrome. J Clin Invest 2006; 116:1784-92

Kalupahana NS, Claycombe K, Newman SJ, Stewart T, Siriwardhana N, Matthan N, Lichtenstein AH, MoustaidMoussa N. Eicosapentaenoic acid prevents and reverses insulin resistance in high-fat diet-induced obese mice via modulation of adipose tissue inflammation. J Nutr 2010;140: 1915-22

Kapur S, Picard F, Perreault M, Deshaies Y, Marette A. Nitric oxide: a new player in the modulation of energy metabolism. Int J Obes Relat Metab Disord 2000;24 Suppl 4:S36-40

Kelly DP, Scarpullar RC. Transcriptional regulatory circuits controlling mitochondrial biogenesis and function. Genes Dev 2004;18:357-68

Kennedy A, Martinez K, Chuang CC, LaPoint K, Mclntosh M. Saturated fatty acid-mediated inflammation and insulin resistance in adipose tissue: mechanisms of action and implications. J Nutr 2009;139:1-4

Kern PA, Di Gregorio GB, Lu T, Rassouli N, Ranganathan G. Adiponectin expression from human adipose tissue: relation to obesity, insulin resistance, and tumor necrosis factoralpha expression. Diabetes 2003;52:1779-85

Koh EH, Park JY, Park HS, Jeon MJ, Ryu JW, Kim M, Kim SY,
Kim MS, Kim SW, Park IS, Youn JH, Lee KU. Essential role of mitochondrial function in adiponectin synthesis in adipocytes. Diabetes 2007;56:2973-81

Koh EH, Kim M, Ranjan KC, Kim HS, Park HS, Oh KS, Park IS, Lee WJ, Kim MS, Park JY, Youn JH, Lee KU. eNOS plays a major role in adiponectin synthesis in adipocytes. Am J Physiol Endocrinol Metab 2010;298:E846-53

Lee HC, Wei YH. Mitochondrial biogenesis and mitochondrial DNA maintenance of mammalian cells under oxidative stress. Int J Biochem Cell Biol 2005;37:822-34

Linscheid $P$, Keller U, Blau N, Schaer DJ, Muller B. Diminished production of nitric oxide synthase cofactor tetrahydrobiopterin by rosiglitazone in adipocytes. Biochem Pharmacol 2003;65:593-8

Liu HR, Tao L, Gao E, Lopez BL, Christopher TA, Willette RN, Ohlstein EH, Yue TL, Ma XL. Anti-apoptotic effects of rosiglitazone in hypercholesterolemic rabbits subjected to myocardial ischemia and reperfusion. Cardiovasc Res 2004;62:135-44

McCall KD, Holliday D, Dickerson E, Wallace B, Schwartz AL, Schwartz C, Lewis CJ, Kohn LD, Schwartz FL. Phenylmethimazole blocks palmitate-mediated induction of inflammatory cytokine pathways in 3T3L1 adipocytes and RAW 264.7 macrophages. J Endocrinol 2010;207:343-53

Nathan C, Xie QW. Nitric oxide synthases: roles, tolls, and controls. Cell 1994;78:915-8

Nisoli E, Clementi E, Paolucci C, Cozzi V, Tonello C, Sciorati C, Bracale R, Valerio A, Francolini M, Moncada S, Carruba MO. Mitochondrial biogenesis in mammals: the role of endogenous nitric oxide. Science 2003;299:896-9

Nozaki M, Fukuhara A, Segawa K, Okuno Y, Abe M, Hosogai $\mathrm{N}$, Matsuda M, Komuro R, Shimomura I. Nitric oxide dysregulates adipocytokine expression in 3T3-L1 adipocytes. Biochem Biophys Res Commun 2007;364:33-9

Perreault M, Marette A. Targeted disruption of inducible nitric oxide synthase protects against obesity-linked insulin resistance in muscle. Nat Med 2001;7:1138-43

Poliandri AH, Machiavelli LI, Quinteros AF, Cabilla JP, Duvilanski BH. Nitric oxide protects the mitochondria of anterior pituitary cells and prevents cadmium-induced cell death by reducing oxidative stress. Free Radic Biol Med 2006;40:679-88

Rachek LI, Musiyenko SI, LeDoux SP, Wilson GL. Palmitate induced mitochondrial deoxyribonucleic acid damage and apoptosis in 16 rat skeletal muscle cells. Endocrinology 2007; 148:293-9

Sato A, Kawano H, Notsu T, Ohta M, Nakakuki M, Mizuquchi $\mathrm{K}$, Itoh M, Suganami T, Ogawa Y. Antiobesity effect of eicosapentaenoic acid in high-fat/high-sucrose diet-induced obesity: importance of hepatic lipogenesis. Diabetes 2010; 59:2495-504

Scherer PE. Adipose tissue: from lipid storage compartment to endocrine organ. Diabetes 2006;55:1537-45

Spiegelman BM. Transcriptional control of mitochondrial energy metabolism through the PGC1 coactivators. Novartis Found Symp 2007;287:60-3 
Tsang MY, Cowie SE, Rabkin SW. Palmitate increases nitric oxide synthase activity that is involved in palmitate-induced cell death in cardiomyocytes. Nitric Oxide 2004;10:11-9

Wang P, Liu J, Li Y, Wu S, Luo J, Yang H, Subbiah R, Chatham J, Zhelyabovska O, Yang Q. Peroxisome proliferator-activated receptor $\{$ delta $\}$ is an essential transcriptional regulator for mitochondrial protection and biogenesis in adult heart. Circ Res 2010;106:911-9

Wenz T, Diaz F, Spiegelman BM, Moraes CT. Activation of the PPAR/PGC-1alpha pathway prevents a bioenergetic deficit and effectively improves a mitochondrial myopathy phenotype. Cell Metab 2008;8:249-56

Wilson-Fritch L, Nicoloro S, Chouinard M, Lazar MA, Chui PC, Leszyk J, Straubhaar J, Czech MP, Corvera S. Mitochondrial remodeling in adipose tissue associated with obesity and treatment with rosiglitazone. J Clin Invest 2004; $114: 1281-9$
Xi L, Qian Z, Xu G, Zhou C, Sun S. Crocetin attenuates palmitate-induced insulin insensitivity and disordered tumor necrosis factor-alpha and adiponectin expression in rat adipocytes. Br J Pharmacol 2007;151:610-7

Yang JY, Koo BS, Kang MK, Rho HW, Sohn HS, Jhee EC, Park JW. Retinoic acid inhibits inducible nitric oxide synthase expression in 3T3-L1 adipocytes. Exp Mol Med 2002;34: 353-60

Yuzefovych L, Wilson G, Rachek L. Different effects of oleate vs. palmitate on mitochondrial function, apoptosis, and insulin signaling in L6 skeletal muscle cells: role of oxidative stress. Am J Physiol Endocrinol Metab 2010;299:E1096E105

Zhou YT, Grayburn P, Karim A, Shimabukuro M, Higa M, Baetens D, Orci L, Unger RH. Lipotoxic heart disease in obese rats: implications for human obesity. Proc Natl Acad Sci USA 2000;97:1784-9 